\title{
Evaluación comparativa de la ac- cesibilidad de los espacios web de las bibliotecas universitarias españolas y norteamericanas
}

\author{
Laura Caballero-Cortés \\ Cristina Faba-Pérez * \\ Félix de Moya-Anegón **
}

Artículo recibido:

2 de octubre de 2008.

Artículo aceptado:

8 de diciembre de 2008.

\section{RESUMEN}

El objetivo principal de la presente investigación es analizar y comparar el grado de cumplimiento de determinadas pautas de accesibilidad web en dos grupos de espacios web que pertenecen a una misma tipología conceptual: "Bibliotecas Universitarias", pero que forman parte de dos realidades geográficas, sociales y económicas diferentes: España y Norteamérica. La interpretación de los resultados pone de manifiesto que es posible utilizar técnicas webmétricas basadas en las características de accesibilidad web para contrastar dos conjuntos de espacios web cerrados.

* Las dos autoras pertenecen a la Universidad de Extremadura, España. (cfabper@alcazaba.unex.es)

** Universidad de Granada, España. felix@ugr.es

INVESTIGACIÓN BIBLIOTECOLÓGICA, Vol. 23, Núm. 47, enero/abril, 2009, México, ISSN: 0187-358X. pp. 45-66 
Palabras clave: Accesibilidad Web, bibliotecas universitarias; España, Norteamérica

\section{ABSTRACT}

Comparative accessibility assessment of Web spaces in Spanish and American university libraries

Laura Caballero-Cortés; Cristina Faba-Pérez and Félix de Moya-Anegón

The main objective of this research is to analyze and compare the degree in which certain Accessibility Guidelines comply with two groups of web spaces which belong to the same conceptual typology: "University Libraries", but conform two different geographic, social and economical realities - Spain and the United States. Interpretation of results reveals the possibility of using web metrics techniques based on Web accessibility characteristics in order to contrast two categories of closed web spaces.

Keywords: Web accessibility; University libraries; Spain; United States

\section{INTRODUCCIÓN}

Omo consecuencia del incremento de los recursos electrónicos y de las dudas de los usuarios finales acerca de la calidad última de ciertos documentos, surge la necesidad de medir y valorar numerosos aspectos de Internet entre los que se encuentra la Accesibilidad de sus recursos. Por ello, desde mediados de la década de 1990, se están aplicando los modelos y métodos informétricos al ámbito de Internet, y más concretamente, a la World Wide Web, y se ha desarrollado un nuevo ámbito de investigación conocido como Cibermetría o Cybermetrics (Cybermetrics, 1997) y Webmetría o Webometrics (Almind e Ingwersen, 1997), respectivamente.

Desde los primeros atisbos de la Web 1.0., el número de páginas, sedes y sitios Web (términos que agrupamos bajo la denominación de "espacios web” —Smith, 1999; Faba-Pérez, Guerrero-Bote y Moya-Anegón, 2003a, 2003b, 2004, 2005-) aumentó de manera espectacular, y la utilización de Internet pasó a formar parte de los intereses comerciales de las empresas para 
darse a conocer mundialmente. En consecuencia, se comenzó a darle mayor importancia a la presentación atractiva del contenido que a la accesibilidad del mismo. Sin embargo, la llegada de la Web 2.0 — también denominada "Web social" y cuyo máximo esplendor se produjo en torno al año 2006más centrada en la participación del usuario y en el desarrollo de servicios y herramientas como la Wikipedia o los Blogs, le concedió a la Accesibilidad Web el lugar que le correspondía.

Según el World Wide Web Consortium (W3C)

hablar de Accesibilidad Web es hablar de un acceso universal a la Web, independientemente del tipo de hardware, software, infraestructura de red, idioma, cultura, localización geográfica y capacidades de los usuarios.

En la actualidad, cuando ya se habla de la Web 3.0. o "Web semántica", la Accesibilidad Web continúa siendo un ámbito muy estudiado y analizado, tanto a nivel general (Bailey y Burd, 2005, 2006; Lazar y Greenidge, 2006) como en campos específicos, como es el caso de las Unidades Informativas Virtuales (Lilly y Van-Fleet, 2000; Golub y Lazić, 2002; López-Marín, Méndez-Rodríguez y Sorli-Rojo, 2002; Acctiva, 2004; Petrie, King y Hamilton, 2005; Yakel, 2005; González-Flórez, 2006) y, en particular, las Bibliotecas Universitarias (Craven, 2000; Schmetzke, 2002, 2003, 2005; Spindler, 2002 Térmens-Graells, Ribera-Turró y Sulé-Duesa, 2002, 2003; Providenti, 2004).

Aunque tradicionalmente la Accesibilidad Web se ha asociado a las personas con discapacidad (Henry, 2002; Casado-Martínez, Mor-Pera y SabatéJordí, 2006), actualmente, debido a los progresos tecnológicos recientes, los problemas de Accesibilidad Web se extienden más allá de los usuarios con discapacidad o con otro tipo de dificultades de acceso, abarcando a la generalidad de los mismos (Tim Berners-Lee, 2000, citado en López-Marín, Méndez-Rodríguez y Sorli-Rojo, 2002; Hassan-Montero y Martín-Fernández, 2004). Por consiguiente, podemos decir que la Accesibilidad Web implica facilitar un acceso universal a la Web, que posibilite su utilización por el mayor número posible de usuarios, independientemente de las discapacidades físicas, sensoriales y cognitivas que presenten, del equipo físico o hardware y de los programas o software que estén usando, y de las condiciones derivadas del contexto de uso.

El presente trabajo analiza y compara el grado de cumplimiento de determinadas Pautas de Accesibilidad Web en dos grupos de espacios web que pertenecen a una misma tipología conceptual: "Bibliotecas Universitarias", pero que forman parte de dos realidades geográficas, sociales y económicas diferentes: "España y Norteamérica", aplicando sobre ellas una tipología de técnicas webmétricas basadas en la Accesibilidad Web. 
La hipótesis de partida para comparar ambos grupos de espacios web es que si se considera que Norteamérica adelanta en tecnología a España, entonces los espacios web de las bibliotecas universitarias norteamericanas deberían cumplir mejor las Pautas de Accesibilidad Web que las españolas (Economist Intelligence Unit, 2006).

\section{MATERIAl y MÉTODO}

El número de espacios web que componen la población de estudio está formado por un total de 188 (66 espacios web de bibliotecas universitarias españolas y 122 de bibliotecas universitarias norteamericanas).

En el caso de España, las fuentes utilizadas para recopilar las bibliotecas universitarias han sido los Directorios electrónicos proporcionados por REBIUN (Red de Bibliotecas Universitarias) y RedIris (Red Académica y de Investigación Nacional). Con respecto a las bibliotecas universitarias norteamericanas, la ARL (Association of Research Libraries) dispone de un listado completo de bibliotecas universitarias y de bibliotecas de instituciones de investigación norteamericanas, de donde se han tomado sus direcciones URL. En ambos casos, la razón principal por la que se han seleccionado dichas fuentes ha sido la oficialidad de las mismas.

La población objeto de estudio (188 espacios web) se ha conformado tras descartar del análisis las bibliotecas universitarias que no disponen de una biblioteca que asuma las funciones de biblioteca central o las que presentan errores de conexión. En total, han sido excluidas once bibliotecas españolas y una norteamericana.

Sobre la población final aplicamos una tipología de técnicas webmétricas basadas en las características de Accesibilidad Web. Estas técnicas usan diversos modelos centrados en estándares, normas o guías que permiten evaluar determinadas características de los espacios web con el fin de comprobar si se puede acceder a ellos independientemente de las circunstancias de las personas que acceden, para lo cual utilizan herramientas de validación automáticas — a veces complementadas con la revisión humana- y esto asegura la objetividad de los resultados.

La herramienta utilizada en este caso para la extracción automática de los errores asociados a las características de Accesibilidad Web de nuestra población ha sido TAW, el Test de Accesibilidad Web desarrollado por la Fundación CTIC (Centro Tecnológico de la Información y de la Comunicación) y financiado por el Ministerio de Industria, Turismo y Comercio de España, y las guías empleadas como modelo de características han sido las "Pautas de 
Accesibilidad al Contenido en la Web 1.0 ( WCAG 1.0)" sobre las que se basa la herramienta TAW para la realización de los análisis. Estas pautas fueron establecidas en 1999 por el World Wide Web Consortium (W3C) a través del grupo de Accesibilidad Web WAI (Web Accesibility Initiative) y actualmente están siendo actualizadas a partir de la publicación —en mayo de 2007del Borrador de Trabajo de Pautas de Accesibilidad al Contenido en la Web 2.0. <http://www.w3.org/TR/WCAG20/>. Las Pautas de Accesibilidad Web (WCAG 1.0) se estructuran en 14 guías divididas en una serie de puntos de verificación, y a su vez, cada punto de verificación tiene asignado uno de los tres niveles de prioridad. Los tres niveles de prioridad establecidos no tienen la misma importancia con respecto al impacto que puede provocar en los diferentes usuarios posibles:

- La prioridad 1 es para los puntos de verificación que el diseñador web "tiene" que satisfacer, y es un requerimiento básico para que algunos grupos de usuarios puedan acceder a la información del documento.

- La prioridad 2 es para los puntos de verificación que el diseñador web "debe" satisfacer, de otra forma, uno o más grupos encontrarán dificultades en acceder a la información del documento. Satisfacer este punto eliminará importantes barreras de acceso a los documentos web.

- La prioridad 3 es para los puntos de verificación que el diseñador web "puede" satisfacer, y aunque puede haber grupos que encuentren alguna dificultad para acceder a la información, satisfacer este punto mejorará la Accesibilidad de los documentos.

Se han considerado los tres niveles de prioridad para la revisión de los conjuntos de espacios web objeto de estudio, además de los problemas de tipo automático y manual detectados por la herramienta TAW. Los informes TAW diferencian los problemas automáticos — que son aquellos que la herramienta TAW tiene la certeza de que incumplen las pautas-, de los problemas manuales - que son los problemas que debería comprobar el desarrollador o diseñador web-.

En el proceso de recolección de los datos se han generado un total de 28 matrices para el global de datos estudiados (en cada matriz, las filas corresponden a los 66 y 122 espacios web de bibliotecas universitarias españolas y norteamericanas, respectivamente, y las columnas de cada una de las 14 guías a los puntos de verificación de cada una de ellas — diferenciando los de tipo manual y automático-).

El procedimiento que hemos usado para hallar los resultados ha sido el cálculo del promedio sobre cada punto de verificación de cada guía en el conjunto 
de los espacios web de las bibliotecas universitarias españolas, por una parte, y en el conjunto de los espacios web de las norteamericanas, por otra. Previamente, para asegurar la objetividad de los resultados, habíamos puesto en relación la vulneración o problemas encontrados en cada punto de verificación de cada biblioteca universitaria con el número de páginas halladas en su espacio web introduciendo, por consiguiente, una variable (el número de páginas) que nos permitía relativizar el número de problemas encontrados en cada biblioteca universitaria teniendo en cuenta su tamaño.

\section{Resultados}

Los resultados obtenidos tras la aplicación de la metodología descrita, se muestran en los gráficos siguientes en los que se representa el promedio y/o el porcentaje del promedio de los puntos de verificación o problemas de Accesibilidad Web que le corresponden a cada conjunto de espacios web proporcionalemente. Se comparan los resultados de las bibliotecas universitarias españolas frente a las norteamericanas en cuanto al incumplimiento de las "Pautas de Accesibilidad al Contenido en la Web 1.0 (WCAG 1.0)". La presentación se realiza a dos niveles: de forma general, comparando los resultados acerca de los problemas de Accesibilidad Web en las tres prioridades y en las guías, y de modo detallado, mostrando los problemas de Accesibilidad concretos de cada una de las guías, puntos de verificación y los problemas de tipo manual y automático en cada prioridad.

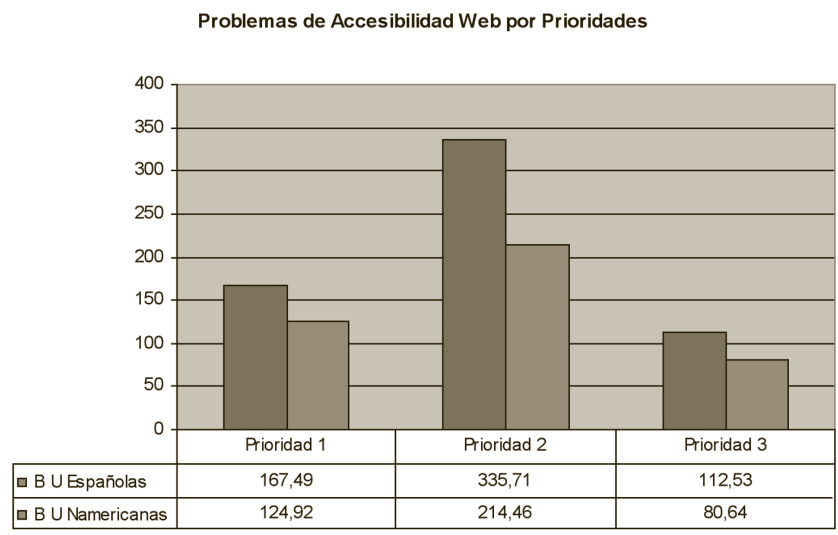

Fig. 1. Problemas de Accesibilidad Web según las Prioridades 
La Figura1 muestra el promedio de los problemas de Accesibilidad Web en los espacios web de las bibliotecas universitarias españolas y norteamericanas según sus prioridades. El promedio total de problemas asciende a 1.035,75 (615,73 para España y 420,02 para Norteamérica, lo que representa un $59 \%$ y un $41 \%$, respectivamente, en la vulneración de características de Accesibilidad Web). Como se observa en la Figura 1, los valores del promedio muestran en ambos grupos de espacios web que los puntos de verificación de prioridad 3 son los que menos se vulneran (podríamos decir que son "los mejores"), seguidos por los puntos de prioridad 1 y finalmente los de prioridad 2. Evidentemente, ya que sería muy difícil hablar de un resultado óptimo (donde no se hubiera violado ningún punto de verificación), lo ideal hubiera sido que el orden en el cumplimiento de las pautas de accesibilidad fuera el de sus prioridades; es decir, la prioridad que debería haberse vulnerado menos debería haber sido la 1 , seguida de la 2 y, en último lugar, la 3.

Problemas de Accesibilidad Web según las Prioridades (porcentual)

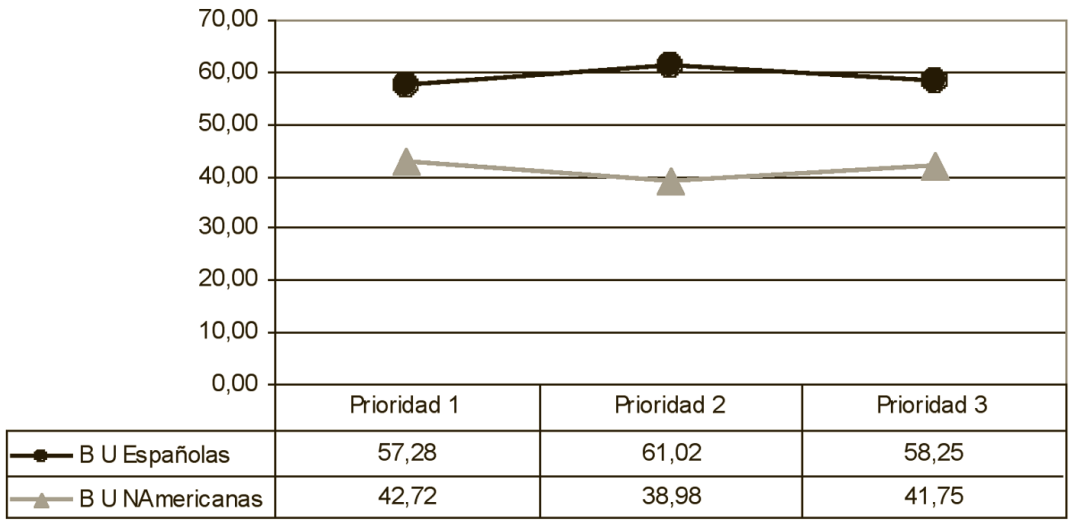

Fig. 2. Porcentaje de problemas de Accesibilidad Web según las Prioridades

En relación a la comparación proporcional entre ambos grupos de bibliotecas en el cumplimiento de las pautas, la Figura 2 muestra que el conjunto español supera proporcionalmente en el número de problemas al conjunto de espacios web de bibliotecas norteamericanas en las tres prioridades, siendo más acusada dicha diferencia negativa para España en el caso de la prioridad 2 (61\% España y $39 \%$ Norteamérica).

Como cada uno de los tres niveles de prioridad está asignado a cada uno de los puntos de verificación de las 14 guías, se presenta la siguiente Tabla 1 y Figura 3 en las que se agrupan, respectivamente, el promedio de problemas y 
los porcentajes proporcionales del promedio de los problemas de ambos conjuntos de bibliotecas según cada guía (denominada "pauta" en las "Pautas de Accesibilidad al Contenido en la Web 1.0").

Tabla 1. Promedio de problemas según las Guías

\begin{tabular}{|c|c|c|}
\hline Guías & B U Españolas & B U NAmericanas \\
\hline G1 & 88,40 & 52,03 \\
\hline G2 & 49,22 & 34,17 \\
\hline G 3 & 99,44 & 61,11 \\
\hline G 4 & 9,40 & 8,07 \\
\hline G 5 & 106,53 & 51,86 \\
\hline G 6 & 51,71 & 43,88 \\
\hline G7 & 3,92 & 8,11 \\
\hline G 8 & 13,31 & 14,90 \\
\hline G9 & 12,38 & 6,89 \\
\hline G10 & 44,31 & 34,66 \\
\hline G11 & 109,81 & 44,54 \\
\hline G12 & 1,35 & 5,65 \\
\hline G13 & 13,76 & 43,47 \\
\hline G14 & 12,18 & 10,68 \\
\hline Total & 615,73 & 420,02 \\
\hline
\end{tabular}

Problemas de Accesibilidad Web según las Guías (porcentual)

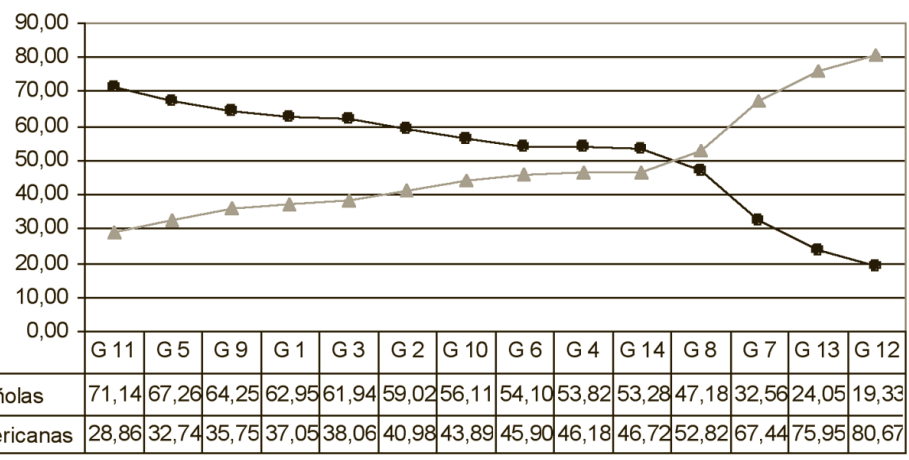

Fig. 3. Porcentaje del promedio de problemas de Accesibilidad Web según las Guías

La Figura 3 nos muestra que, en general, las bibliotecas universitarias españolas tienen un porcentaje mayor de problemas que las norteamericanas, excepto en las guías 8, 7, 13 y 12, relacionadas con la accesibilidad directa de las interfaces incrustadas (guía 8), con el control sobre los cambios de los contenidos tempo-dependientes (guía 7), con la información de contexto y 
orientación (guía 13) y con los mecanismos de navegación (guía 12). Como podemos observar, en la guía 8 las bibliotecas universitarias españolas representan el 47,18\% de los problemas, en la guía 7 el 32,56\%, en la guía 13 el $24,05 \%$ y en la guía 12 el 19,33\% de los mismos. Por el contrario, las guías más vulneradas por los espacios web de bibliotecas universitarias españolas son la $11(71,14 \%)$ (relacionada con el uso de las tecnologías y pautas WC3) y la $5(67,26 \%)$ (que se relaciona con la creación de tablas). Aunque podríamos considerar desafortunado el hecho de que el conjunto español mejore al norteamericano solo en guías cuyos problemas no son prácticamente de prioridad 1 (de los 20 puntos de verificación que acumulan las guías 7, 8, 12 y 13, sólo 3 puntos son de prioridad 1, lo que supone el 15\%), hay que tener en cuenta que las guías que incumple de manera significativa (11 y 5) afortunadamente tampoco presentan un porcentaje demasiado elevado de puntos de verificación de prioridad 1 (30\%).

A modo de sinopsis podemos decir que de las 14 guías, el conjunto de bibliotecas españolas, proporcionalmente, mejora al conjunto norteamericano en 4 (guías 7, 8, 12 y 13) (puesto que presenta porcentajes $<50 \%$ respecto a las bibliotecas norteamericanas), mientras que el conjunto norteamericano mejora al español en 10 guías.

La Tabla 2 y la Figura 4 muestran, respectivamente, el promedio de problemas y los porcentajes del promedio de problemas de ambos conjuntos de bibliotecas en la prioridad 1 .

Tabla 2. Promedio de problemas en la Prioridad 1

\begin{tabular}{|c|c|c|c|c|}
\hline \multicolumn{5}{|c|}{ Prioridad 1} \\
\hline $\begin{array}{c}\text { Manual/ } \\
\text { Automático }\end{array}$ & Guías & P.Verificación & BUEspañolas & BUNAmericanas \\
\hline M & 1 & 1.1. & 49,08 & 47,09 \\
\hline$A$ & 1 & 1.1. & 38,84 & 4,57 \\
\hline \multirow[t]{9}{*}{$\mathrm{M}$} & 1 & 1.2 & 0,08 & 0,0003 \\
\hline & 1 & 1.3. & 0,00 & 0,000007 \\
\hline & 1 & 1.4 & 0,00 & 0,000007 \\
\hline & 2 & 2.1. & 1,31 & 2,70 \\
\hline & 4 & 4.1. & 1,31 & 2,70 \\
\hline & 5 & 5.1. & 14,23 & 8,67 \\
\hline & 5 & 5.2. & 14,28 & 8,67 \\
\hline & 6 & 6.1. & 19,49 & 12,28 \\
\hline & 6 & 6.2. & 5,41 & 7,45 \\
\hline$A$ & 6 & 6.2 & 0,04 & 0,00 \\
\hline$M$ & 6 & 6.3. & 12,79 & 14,77 \\
\hline$A$ & 6 & 6.3. & 0,00 & 0,46 \\
\hline
\end{tabular}




\begin{tabular}{|c|c|c|c|c|}
\hline M & 7 & 7.1. & 1,31 & 2,70 \\
\cline { 2 - 5 } & 8 & $8.1 . \mathrm{a}$ & 6,65 & 7,45 \\
\cline { 2 - 5 } & 9 & 9.1. & 0,08 & 0,00004 \\
\cline { 2 - 5 } & 11 & 11.4. & 1,31 & 2,70 \\
\cline { 2 - 5 } & 12 & 12.1. & 0,00 & 0,00 \\
\cline { 2 - 5 } & 14 & 14.1. & 1,31 & 2,70 \\
\hline Total & 11 & 20 & 167,49 & 124,92 \\
\hline
\end{tabular}

La Figura 4 (página siguiente) representa el porcentaje del promedio de problemas de prioridad 1 para ambos conjuntos de datos. Como se observa, el grupo de bibliotecas españolas vulnera en mayor proporción que las norteamericanas los puntos de verificación 6.2., 1.1. (automáticos) y 9.1., 1.2., 5.2., 5.1. 6.1. y 1.1. (manuales). En este último elemento de verificación 1.1. (manual) se produce prácticamente un punto de inflexión y los espacios web de las bibliotecas españolas comienzan a incumplir las pautas de prioridad 1 en menor proporción que las norteamericanas. En este punto 1.1. (manual) los porcentajes de vulneración son muy próximos en ambos conjuntos $(51,03 \%$ para España y 48,97\% para Norteamérica). Las consecuencias de su incumplimiento son que los lectores de pantalla, así como los navegadores "sólo texto", no ofrecen información sobre las imágenes (puesto que no despliegan gráficos) y por lo tanto, es importante que el código fuente ofrezca alternativas textuales para cualquier tipo de gráfico que actúe o sirva como enlace.

Destacan especialmente los puntos de verificación 1.3., 1.4. (problemas manuales) y 6.3. (automático) vulnerados por los espacios web de las bibliotecas universitarias norteamericanas pero no por las españolas. Los dos primeros puntos son problemas de tipo manual y afectan a las presentaciones multimedia, y el punto 6.3. exige que las páginas sigan siendo utilizables cuando se desconecten o no soporten los scripts, applets u otros objetos programados. En contraposición, también sucede a la inversa, ya que los espacios web de las bibliotecas españolas incumplen el punto de verificación 6.2 (de tipo automático, relacionado con los contenidos dinámicos) pero no así las norteamericanas.

El aspecto más significativo es que la guía 12 en el punto de verificación 12.1. manual (que obliga a ponerle título a cada marco para facilitar su identificación y navegación) no es vulnerado por ninguno de los dos conjuntos de espacios web, debido a que es una práctica muy extendida para el diseño de páginas web. Las guías 3, 10 y 13 no son representadas porque en la prioridad 1 no tienen asignados puntos de verificación (lo que significa que estas guías no son un requerimiento básico para el cumplimiento de las Pautas de Accesibilidad (WCAG 1.0)). 


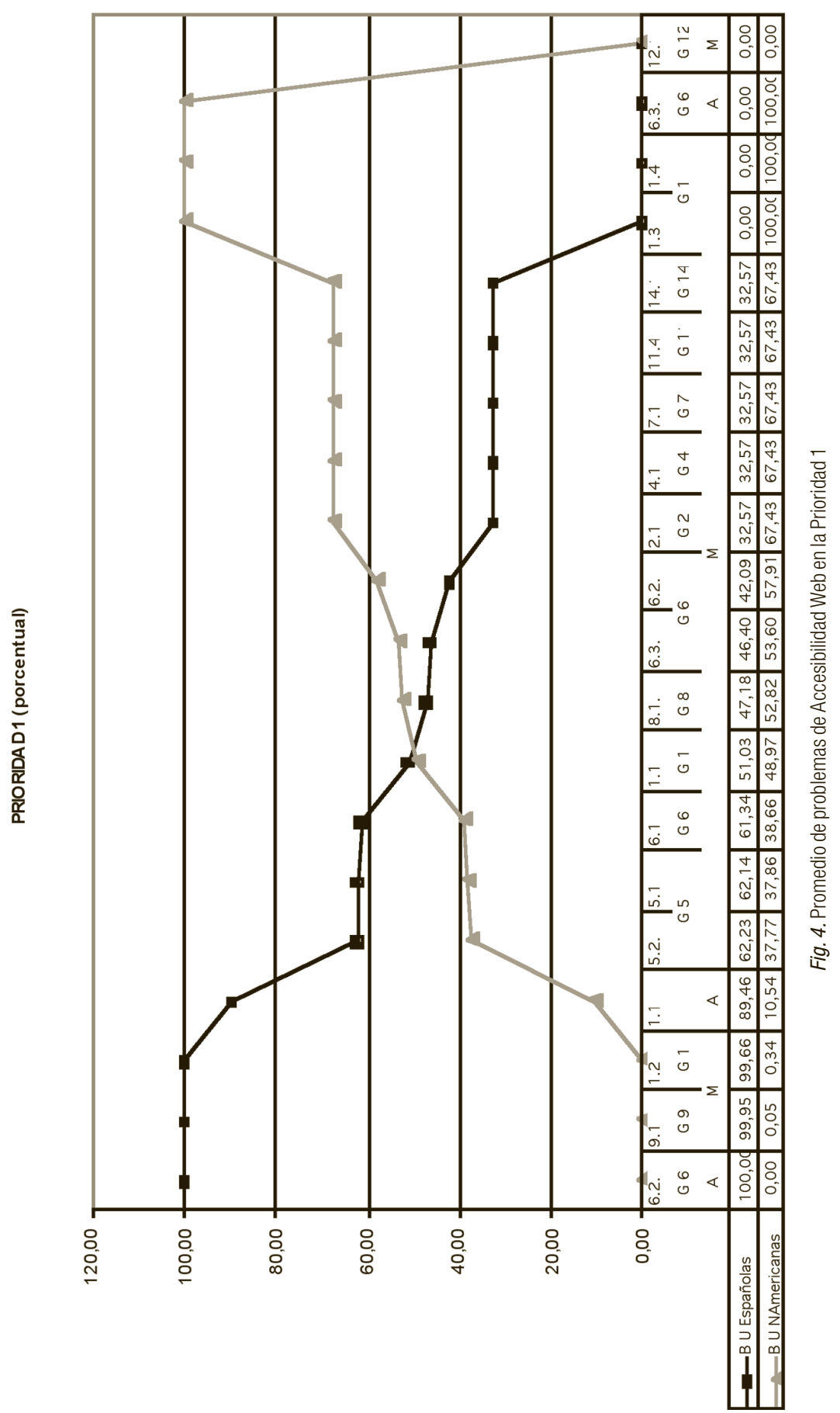


A modo de sinopsis con respecto a la prioridad 1, podemos decir que de los 19 puntos de verificación manuales y automáticos que se incumplen en la prioridad 1 (todos excepto el 12.1.) el conjunto de bibliotecas españolas, proporcionalmente, mejora al conjunto norteamericano en 11 de los 19 puntos de verificación de la prioridad 1 (puesto que presenta porcentajes $<50 \%$ respecto a las bibliotecas norteamericanas), mientras que el conjunto norteamericano mejora al español en 8 puntos de verificación. Ahora bien, si nos fijamos en los promedios no porcentuales (Tabla 2), el número de problemas totales del conjunto español en las guías en la prioridad 1 es mayor $(167,49)$ que el norteamericano $(124,92)$.

La Tabla 3 y la Figura 5 muestran, respectivamente, el promedio de problemas y los porcentajes del promedio de problemas de ambos conjuntos de bibliotecas en la prioridad 2 .

Tabla 3. Promedio de problemas en la Prioridad 2

\begin{tabular}{|c|c|c|c|c|}
\hline \multicolumn{5}{|c|}{ Prioridad 2} \\
\hline $\begin{array}{c}\text { Manual/ } \\
\text { Automático }\end{array}$ & Guía & P.Verificación & BU Españolas & B UNAmericanas \\
\hline$M$ & 2 & 2.2. a & 46,60 & 28,76 \\
\hline \multirow[t]{6}{*}{$A$} & 3 & 3.2. & 0,51 & 0,026 \\
\hline & 3 & 3.3. & 0,25 & 0,021 \\
\hline & 3 & 3.4 & 34,47 & 20,41 \\
\hline & 3 & 3.5. & 1,17 & 1,91 \\
\hline & 3 & 3.6. & 0,006 & 0,001 \\
\hline & 3 & 3.7. & 0,01 & 0,00 \\
\hline \multirow[t]{11}{*}{$M$} & 3 & 3.1. & 1,19 & 2,70 \\
\hline & 3 & 3.2 & 0,79 & 2,68 \\
\hline & 3 & 3.3. & 47,73 & 20,82 \\
\hline & 3 & 3.4 & 9,57 & 5,27 \\
\hline & 3 & 3.5. & 0,59 & 1,74 \\
\hline & 3 & 3.6. & 1,30 & 2,70 \\
\hline & 3 & 3.7. & 1,84 & 2,82 \\
\hline & 5 & 5.3. & 32,51 & 12,97 \\
\hline & 5 & 5.4 & 0,002 & 0,003 \\
\hline & 6 & 6.4 & 13,98 & 8,92 \\
\hline & 6 & 6.5 & 0,00 & 0,00 \\
\hline A & 7 & 7.3. & 0,04 & 0,00 \\
\hline \multirow[t]{4}{*}{$M$} & 7 & 7.2 & 1,31 & 2,70 \\
\hline & 7 & 7.3. & 1,26 & 2,70 \\
\hline & 7 & 7.4 & 0,00 & 0,00 \\
\hline & 7 & 7.5 & 0,00 & 0,00 \\
\hline
\end{tabular}




\begin{tabular}{|c|c|c|c|c|}
\hline \multirow{4}{*}{ M } & 8 & $8.1 . b$ & 6,65 & 7,45 \\
\cline { 2 - 5 } & 9 & 9.2. & 2,48 & 0,002 \\
\cline { 2 - 5 } & 9 & 9.3. & 7,39 & 1,51 \\
\cline { 2 - 5 } & 10 & 10.1. & 8,28 & 13,23 \\
\cline { 2 - 5 } & 10 & 10.2. & 0,39 & 3,17 \\
\hline $\mathrm{A}$ & 11 & 11.2. & 105,89 & 36,43 \\
\hline $\mathrm{M}$ & 11 & 11.1. & 1,31 & 2,70 \\
\cline { 2 - 5 } & 12 & 12.2. & 0,00 & 0,00 \\
\hline \multirow{3}{*}{$\mathrm{A}$} & 12 & 12.3. & 0,25 & 0,83 \\
\cline { 2 - 5 } & 12 & 12.4. & 1,09 & 3,95 \\
\hline \multirow{3}{*}{$\mathrm{M}$} & 12 & 12.3. & 0,02 & 0,87 \\
\cline { 2 - 5 } & 13 & 13.1. & 2,89 & 2,70 \\
\cline { 2 - 5 } & 13 & 13.2. & 1,31 & 2,70 \\
\cline { 2 - 5 } & 13 & 13.3. & 1,30 & 2,70 \\
\cline { 2 - 5 } & 13 & 13.4. & 335,71 & 214,46 \\
\hline
\end{tabular}

Destaca de la Tabla 3, la gran cantidad de puntos de verificación incumplidos por ambos conjuntos de espacios web de las bibliotecas universitarias - un total de 34 puntos de los 38 - aunque con valores relativamente bajos, exceptuando el punto de verificación 11.2. (automático) "Evite características desaconsejadas por las tecnologías W3C (World Wide Web Consortium)" vulnerado de forma espectacular por los espacios web de las bibliotecas universitarias españolas con un promedio de 105,89 problemas de Accesibilidad Web, y también con el máximo valor de incumplimiento por parte de las americanas con un promedio de 36,43 problemas (representado proporcionalmente en porcentajes de $74,4 \%$ y de $25,65 \%$, respectivamente). 

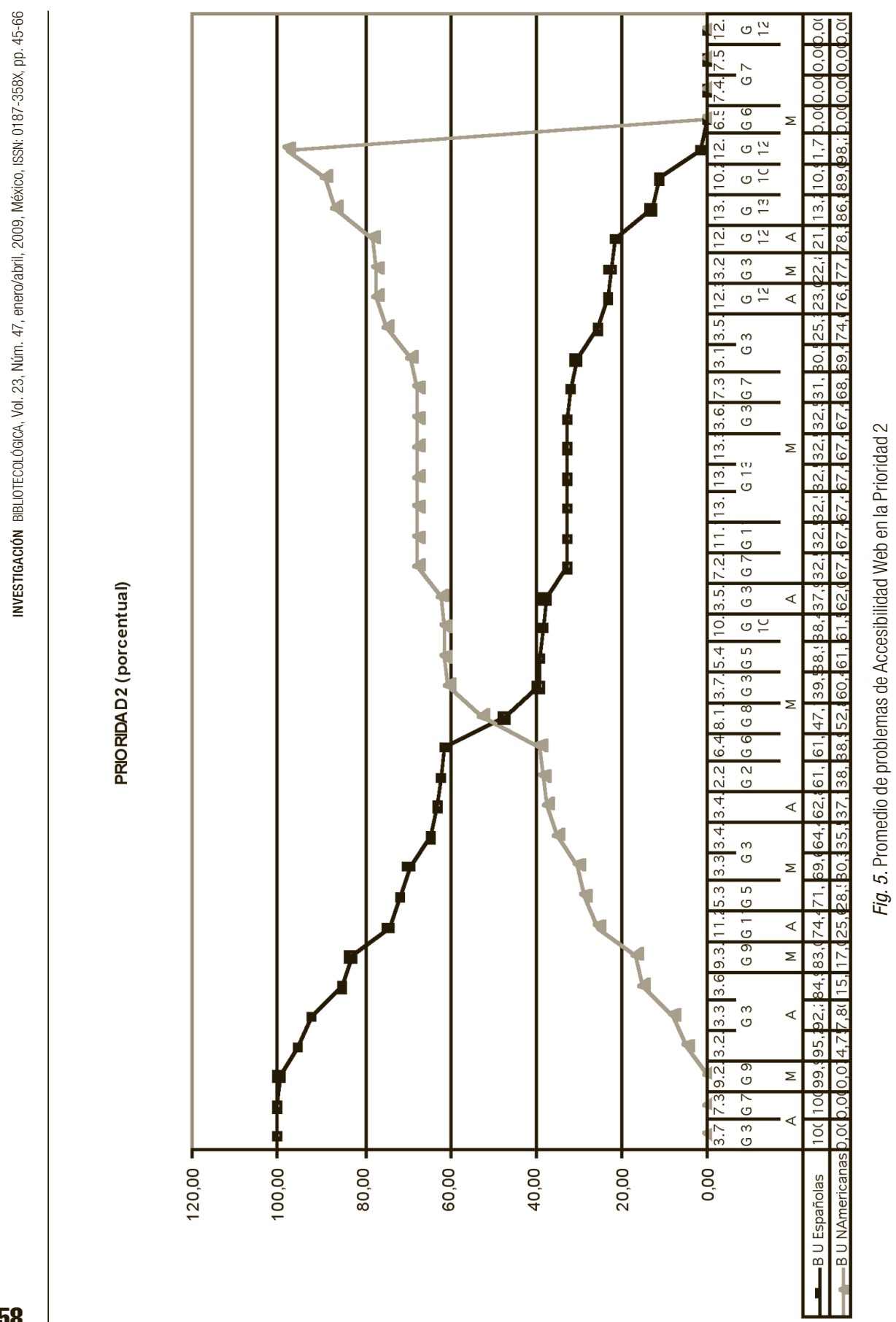
En la Figura 5 (página anterior) observamos que los espacios web de las bibliotecas universitarias norteamericanas presentan un mayor grado de incumplimiento respecto a las españolas en 20 puntos de verificación, con porcentajes que oscilan entre el 98,23\% (12.3. manual) y el 52,82\% (8.1.b. manual). En el caso contrario, los espacios web las bibliotecas universitarias españolas tienen un porcentaje mayor de problemas respecto a las norteamericanas en 14 puntos de verificación cuyos porcentajes oscilan entre el 61,04\% (6.4. manual) y el 100\% (3.7. y 7.3. automático).

En esta prioridad 2 se vuelven a localizar problemas que tienen los espacios web españoles pero no los norteamericanos, sin embargo, esta vez no sucede a la inversa. Así, en los puntos de verificación 3.7. y 7.3. (automáticos), las bibliotecas españolas no cumplen las Pautas de Accesibilidad (WCAG 1.0) que especifican "Marque las citas. No utilice el marcador de citas para efectos de formato tales como sangrías", y "Hasta que las aplicaciones de usuario permitan congelar el movimiento de los contenidos, evite los movimientos en las páginas", respectivamente.

En contraposición a todos estos problemas de Accesibilidad Web, los puntos de verificación no vulnerados por ambos conjuntos de espacios web son 6.5., 7.4, 7.5. y 12.2. (manuales). Por tanto, se puede interpretar que se cumplen los puntos relacionados con la accesibilidad de los contenidos dinámicos (6.5.), con la actualización automáticamente de los objetos y las páginas (7.4. y 7.5.) o con la descripción de marcos (12.2.). Con respecto a las guías 1, 4 y 14 no son representadas porque no tienen puntos de verificación en la prioridad 2 .

Para finalizar con esta prioridad 2, podemos decir que de los 34 puntos de verificación manuales y automáticos que se incumplen en la prioridad 2, el conjunto de bibliotecas españolas, proporcionalmente, mejora al conjunto norteamericano en 20 puntos de verificación (puesto que presenta porcentajes $<50 \%$ respecto a las bibliotecas norteamericanas), mientras que el conjunto norteamericano mejora al español en 14 puntos de verificación. No obstante, si nos fijamos en los promedios no porcentuales (Tabla 3), el número de problemas totales del conjunto español en las guías en la prioridad 2 es mayor $(335,71)$ que el norteamericano $(214,46)$.

La Tabla 4 y la Figura 6 muestran, respectivamente, el promedio de problemas y los porcentajes proporcionales del promedio de problemas de ambos grupos estudiados en la prioridad 3 . 
Tabla 4. Promedio de problemas en la Prioridad 3

\begin{tabular}{|c|c|c|c|c|}
\hline \multicolumn{5}{|c|}{ Prioridad 3} \\
\hline $\begin{array}{c}\text { Manual/ } \\
\text { Automático }\end{array}$ & Guía & P.Verificación & BU Españolas & B U NAmericanas \\
\hline$A$ & 1 & 1.5 . & 0,40 & 0,36 \\
\hline M & 2 & 2.2. b & 1,31 & 2,70 \\
\hline \multirow[t]{2}{*}{ A } & 4 & 4.2 . & 0,0011 & 0,0009 \\
\hline & 4 & 4.3. & 6,79 & 2,66 \\
\hline$M$ & 4 & 4.2 . & 1,31 & 2,70 \\
\hline \multirow[t]{2}{*}{ A } & 5 & 5.5 . & 31,24 & 12,95 \\
\hline & 5 & 5.6 . & 0,001 & 0,0005 \\
\hline \multirow[t]{3}{*}{$M$} & 5 & 5.5. & 14,27 & 8,60 \\
\hline & 9 & 9.4 . & 1,28 & 2,70 \\
\hline & 9 & 9.5. & 1,15 & 2,67 \\
\hline A & 10 & 10.4. & 0,45 & 3,00 \\
\hline \multirow[t]{11}{*}{ M } & 10 & 10.3. & 32,51 & 12,97 \\
\hline & 10 & 10.5. & 2,69 & 2,30 \\
\hline & 11 & 11.3. & 1,31 & 2,70 \\
\hline & 13 & 13.5. & 1,31 & 2,70 \\
\hline & 13 & 13.6. & 1,31 & 2,70 \\
\hline & 13 & 13.7 & 0,47 & 2,81 \\
\hline & 13 & 13.8 & 1,31 & 2,70 \\
\hline & 13 & 13.9 & 1,26 & 2,70 \\
\hline & 13 & 13.10 & 1,31 & 2,70 \\
\hline & 14 & 14.2. & 1,31 & 2,70 \\
\hline & 14 & 14.3. & 9,57 & 5,27 \\
\hline Total & 9 & 22 & 112,53 & 80,64 \\
\hline
\end{tabular}

Como se representa en la Tabla 4, el nivel de incumplimiento más elevado en ambos conjuntos de bibliotecas universitarias se produce en los puntos de verificación 5.5. (automático) y 10.3. (manual). El punto 5.5. hace referencia al suministro de resúmenes de las tablas y el 10.3. señala la necesidad de proporcionar un texto lineal alternativo para todas las tablas hasta que las aplicaciones de usuario interpreten correctamente los textos contiguos.

La Figura 6 (página siguiente) nos muestra que si comparamos el conjunto de espacios web de bibliotecas universitarias españolas y norteamericanas en el cumplimiento de los puntos de verificación de la prioridad 3, observamos que éstas últimas incumplen en mayor proporción 13 puntos de verificación cuyos porcentajes oscilan entre el 87,05\% (10.4 automático) y el 67,42\% (4.2. manual) de vulneración respecto al conjunto español. En el caso de los webs españoles, son 9 los puntos de verificación que incumplen en mayor pro- 


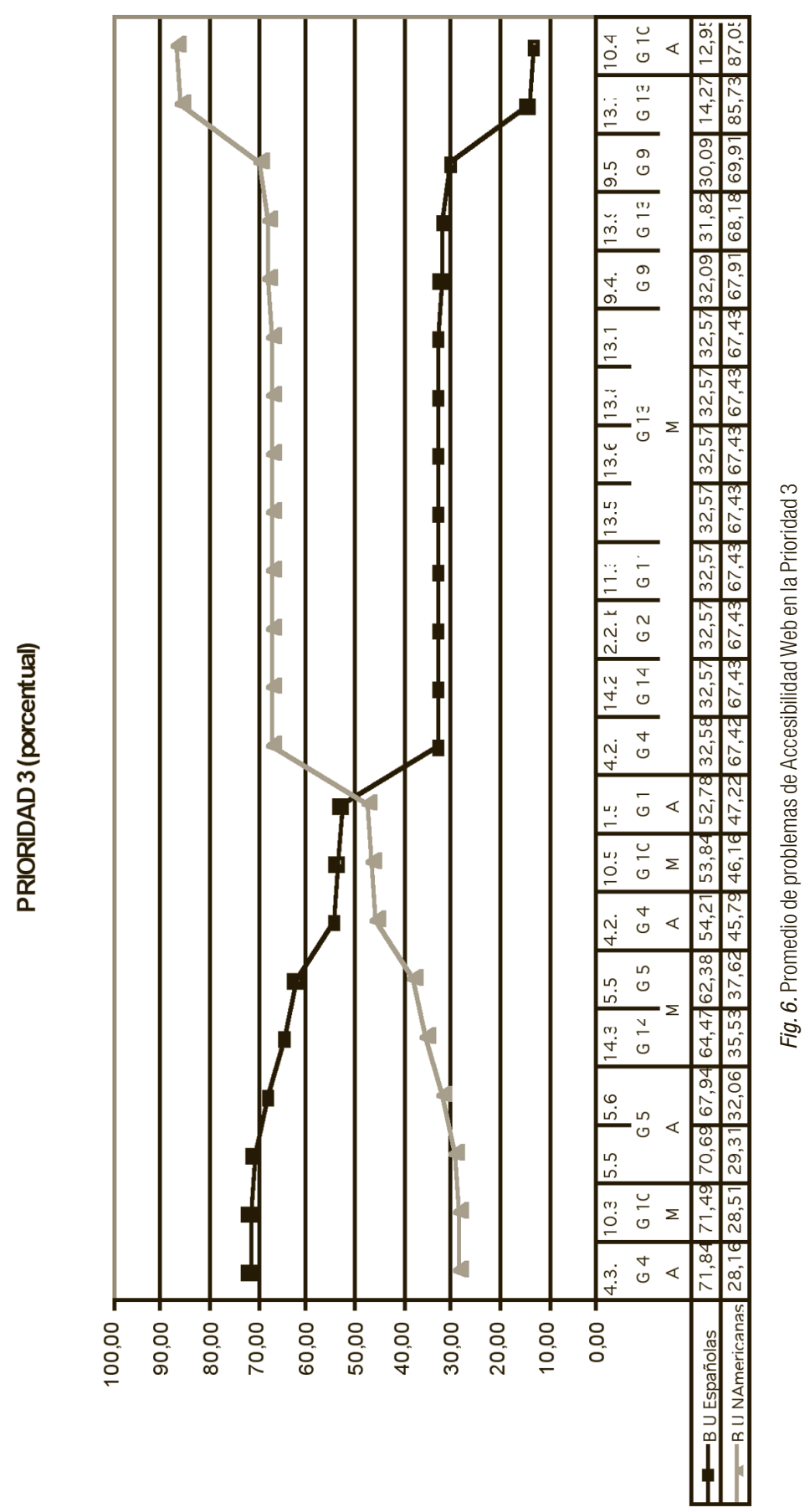


porción respecto a los norteamericanos con porcentajes que fluctúan entre el 71,84\% (4.3. automático) y el 52,78\% (1.5. automático).

Aunque en el caso de la prioridad 3 no existen puntos de verificación que no hayan sido vulnerados por alguno de los dos conjuntos de bibliotecas, no es tan grave porque estamos hablando de la prioridad con el nivel más bajo de preferencia en su cumplimiento. Asimismo, las guías 3, 6, 7, 8 y 12 no han sido representadas porque no tienen puntos de verificación en esta prioridad.

Para resumir podemos decir que los 22 puntos de verificación manuales y automáticos se incumplen en la prioridad 3. El conjunto de bibliotecas españolas mejora, proporcionalmente, al conjunto norteamericano en 13 puntos de verificación de la prioridad 3 (puesto que presenta porcentajes $<50 \%$ respecto a las bibliotecas norteamericanas), mientras que el conjunto norteamericano mejora al español en 9 puntos de verificación. De cualquier forma, los promedios no porcentuales de la Tabla 4 nos muestran que el número de problemas totales del conjunto español en las guías en la prioridad 3 es mayor $(112,53)$ que el norteamericano $(80,64)$.

\section{Conclusiones}

Los resultados obtenidos y su posterior interpretación nos han mostrado que ambos conjuntos vulneran las pautas de Accesibilidad Web (España con un $59 \%$ y Norteamérica con un $41 \%$ del total), lo que nos permite una primera conclusión general: los espacios web de las bibliotecas universitarias españolas, si bien incumplen en mayor proporción las pautas de accesibilidad (corroborando la hipótesis de partida), no se encuentran a una gran distancia del conjunto norteamericano. De hecho, la diferencia no resulta estadísticamente significativa como para evidenciar de forma clara una política editorial orientada visiblemente a la accesibilidad por parte de los webs norteamericanos, frente a la política contraria por parte de los espacios web españoles.

\section{- Cumplimiento de las Prioridades}

En ambos conjuntos se infringen las tres prioridades en el mismo orden de vulneración de menor a mayor (prioridad 3, 1 y 2) y en proporciones muy similares (prioridad 3: España 18,28\%, Norteamérica 19,20\% ; prioridad 1: España 27,20\%, Norteamérica 29,74\% y prioridad 2: España 54,52\%, Norteamérica 51,05\%). Podemos concluir que ambos grupos cumplen mejor las pautas que no son de obligado cumplimiento (prioridad 3). 
- Cumplimiento de las Guías

De las 14 guías, el conjunto de bibliotecas españolas, vulnera en menor proporción que el norteamericano sólo las guías 7, 8, 12 y 13, lo que nos permite concluir que en aspectos no relacionados con la prioridad 1 (es decir, no especialmente significativos: como el control sobre los cambios de los contenidos tempo-dependientes, la accesibilidad directa de las interfaces incrustadas, la información de contexto o los mecanismos de navegación), España se halla mejor situada que el conjunto norteamericano.

- Cumplimiento de los puntos de verificación

De los 75 puntos de verificación infringidos en las tres prioridades, el conjunto de bibliotecas españolas vulnera en menor proporción que el norteamericano 44 puntos (que suman 81,68 errores de los 615,73 infringidos en total por el conjunto español -13,27\%-), mientras que el conjunto norteamericano vulnera en menor proporción 31 puntos (que suman 161,55 errores de los 420,02 infringidos en total por el grupo norteamericano -38,46\%-). Aunque podríamos concluir que esta situación es beneficiosa para el conjunto español, los puntos de verificación que España vulnera en mayor proporción que Norteamérica, aunque son menos, son infringidos con gran peso $(86,73 \%)$.

La confirmación de la hipótesis de partida:

si se considera que Norteamérica adelanta en tecnología a España, entonces los espacios web de las bibliotecas universitarias norteamericanas deberían cumplir mejor las Pautas de Accesibilidad Web que las españolas

nos permite concluir que los avances tecnológicos y económicos de Norteamérica facilitan un acceso universal a la Web, posibilitando su utilización por el mayor número posible de usuarios, independientemente de cualquier circunstancia. No obstante, las bibliotecas universitarias virtuales de España no se hallan especialmente distantes de las norteamericanas en cuanto a favorecer el acceso universal a la Web, mejorándolas en aspectos como el control sobre los cambios de los contenidos tempo-dependientes, la accesibilidad directa de las interfaces incrustadas, la información de contexto o los mecanismos de navegación. No obstante, en el caso de las bibliotecas norteamericanas, sí parece haber una mayor preocupación por satisfacer ciertos estándares de diseño (lo que quizá podría explicarse por un mayor grado de participación de empresas y profesionales del mundo del diseño en el caso de estas bibliotecas. En definitiva, un mayor nivel de profesionalización de los diseños). 


\section{BiBLIOGRAFÍA}

ACCTIVA (2004), Breve comparativa de la Usabilidad y Accesibilidad de los Portales de los Museos Estatales. Disponible en: http:// www.acctiva.com/recursos/AcctivaMuseos.pdf (visitada: 10 Septiembre 2007).

Almind, T. C., Ingwersen, P. (1997), "Informetric analyses on the World Wide Web: methodological approaches to "Webometrics", en Journal of Documentation, 53 (4), 404-426.

Bailey, J., Burd, E. (2005), "Web accessibility evolution in the United Kingdom”, en Seventh IEEE International Symposium on Web Site Evolution (WSE 2005), pp. 79-84.

(2006), "What is the current state of Web Accessibility?", en Eighth IEEE International Symposium on Web Site Evolution (WSE 2006), pp. 69-74.

Berners-Lee, T., (2000), Tejiendo la red: inventor del World Wide Web nos descubre su origen, Madrid,, Siglo Veintiuno.

Casado-Martínez, C., Mor-Pera, E., Sabaté-Jordí, Ll., (2006), "Accesibilidad en la Web”, en Mosaic, (47), disponible en: http://mosaic.uoc. edu/articulos/accesibilidad0306.html (visitada: 24 Agosto 2007).

Craven, J. (2000), "Electronic Access for All: Awareness in Creating Accessible Web Sites for the University Library", en Disability and Information Systems in Higher Education (DISinHE), disponible en: http://www.dmag.org.uk/resources/casestudies/cravenfull.asp (visitada: 9 Septiembre 2007).

CYBERMETRICS, International Journal of Scientometrics, Informetrics and Bibliometrics (1997), disponible en: http://www.cindoc.csic. es/cybermetrics/ (visitada: 6 Febrero 2007).

ECONOMIST INTELLIGENCE UNIT (2006), The 2006 e-readiness rankings: A white paper from the Economist Intelligence Unit, disponible en: http://www-935.ibm.com/services/us/gbs/bus/pdf/ ibv-2006-e-readiness-webrr.pdf (visitada: 15 Agosto 2007).

Faba-Pérez, C., Guerrero-Bote, V. P., Moya-Anegón, F. (2003a), "Data Mining in a Closed Web Environment”, en Scientometrics, 58(3), 623-640.

(2003b), " 'Sitation' distributions and Bradford's Law in a closed web space", en Journal of Documentation, 59(5), 558-580. (2004), "Methods for analysing web citations: a study of web-coupling in a closed environment", en Libri, 54(1), 43-53.

(2005), "Self-Organizing Maps in Web Spaces based in Formal Characteristics", en Information Processing \& Management, 41, 331-346.

Golub, K., Lazić, N. (2002), “Accessibility of public library Web sites”, en Libraries in the digital age, Dubrovnik, 2002: Integrating information seeking and information services - practice and research, disponible en: http://www.it.lth.se/koraljka/Lund/publ/GolubLazicLIDA2002-eng.pdf (visitada: 18 Agosto 2007). 
González-Flórez J. A. (2006), Pautas de accesibilidad web para bibliotecas. Buenos Aires, Alfagrama.

Hassan-Montero, y., Martín-Fernández, F. J. (2004), "Propuesta de adaptación de la metodología de diseño centrado en el usuario para el desarrollo de sitios web accesibles", en Revista Española de Documentación Cientifica, 27 (3), 330-345.

Henry, S.L. (2002), "Another-ability: Accessibility Primer for Usability Specialists”, en UPA 2002, the Usability Professionals' Association Annual Conference, disponible en: http://www.uiaccess.com/upa2002a.html (visitada: 28 Junio 2007).

Lazar, J. y Greenidge, K. D. (2006), “One year older, but not necessarily wiser: an evaluation of homepage accessibility problems over time”, en Universal Access in the Information Society, 4 (4) : 285291.

Lilly, E. B., Van-Fleet, C. (2000), "Measuring the accessibility of public library home pages", en Reference E User Services Quarterly, 40 (2), 156-165.

López-Marín, L., Méndez-Rodríguez, E. M., Sorli-Rojo, A. (2002), "Evaluación de la accesibilidad y usabilidad de los sitios web de las bibliotecas públicas catalanas”, en Item: Revista de biblioteconomía i documentació, (31), 17-51.

Petrie, H., King, N., Hamilton, F. (2005), Museums, Libraries and Archives Council. Accessibility of museum, library and archive websites: the MLA audit, London, City University, disponible en: http://www.mla.gov.uk/resources/assets//M/mla_web_accessibility_pdf_6541.pdf (visitada: 1 septiembre 2007).

Providenti, M. (2004), "Library Web Accessibility at Kentucky's 4year Degree Granting Colleges and Universities”, en D-Lib Magazine, 10 (9), disponible en: http://www.dlib.org/dlib/september04/providenti/09providenti.html (visitada: 24 Agosto 2007).

Schmetzke, A. (2003), "Web Accessibility at University Libraries and Library Schools: 2002 Follow-up Study”, en M. HRICKO (Ed.), Design and Implementation of Web-Enabled Teaching Tools. Hershey, PA, Idea Group Publishing, pp. 145-189.

(2002), Web page accessibility at 56 North American campuses: 2002 Survey data on libraries and library schools, disponible en: http://library.uwsp.edu/aschmetz/Accessible/nationwide/ Survey2002/contents2002.htm (visitada: 9 Septiembre 2007).

(2005), "Web Accessibility at University of Wisconsin Libraries", en Wisconsin Association of Academic Librarians (WAAL), Newsletter, 22 (3), 5-9, disponible en: http://www.wla.lib.wi.us/ waal/newsletter/223.html\#webaccess (visitada: 9 Septiembre de 2007).

Smith, A. G. (1999), "The impact of web sites: a comparison betwen Australasia an Latin America”, en INFO'99, Congreso Internacional de Información, disponible en: http://www.vuw.ac.nz/ agsmith/ publns/austlat/ (visitada: 12 Mayo 2006). 
Spindler, T. (2002), "The Accessibility of Web Pages for Mid-sized College and University Libraries", en Reference y User Services Quarterly, 42 (2), 149-154, disponible en: http://digitalcommons. rwu.edu/cgi/viewcontent.cgi? article=1000ycontext=librarypub (visitada: 15 Julio 2007).

Térmens-Graells, M., Ribera-Turró, M., Sulé-Duesa, A. (2002), "L'accessibilitat de les webs de les universitats catalanes", en Item: Revista de biblioteconomía i documentació, (31), 53-76.

(2003), "Nivel de accesibilidad de las sedes web de las universidades Españolas", en Revista Española de Documentación Cientifica, 26 (1), 21-39.

Yakel, E. (2005), "Los archivos en la era de la accesibilidad", en Lligall. Revista Catalana d'Arxivística, 23, 117-134. 